\title{
Link between obesity and COVID-19 may not be what it seems
}

\author{
- Cite as: CMAJ 2021 July 19;193:E1096-7. doi: 10.1503/cmaj.1095952
}

Posted on cmajnews.com on June 25, 2021.

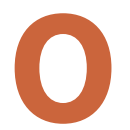

besity is frequently cited as a risk factor for severe COVID19 , but the role of weight bias and discrimination in that risk demands further investigation, according to Dr. Yoni Freedhoff, an associate professor of family medicine at the University of Ottawa and medical director of the Bariatric Medical Institute in Ottawa.

"Are these outcomes truly a consequence of physiology? Or is there something else at play?" Freedhoff questioned at the 2021 Canadian Obesity Summit.

There is no doubt that people with higher body mass index (BMI) suffer worse outcomes from COVID-19. One meta-analysis that pooled data on more than 399000 people with COVID-19 found that those with obesity were $113 \%$ more likely to be hospitalized, $74 \%$ more likely to need intensive care and $48 \%$ more likely to die than those with lower BMIs.

Researchers have attributed this increased risk to a constellation of physiological factors - from weakened immune responses due to chronic inflammation, to breathing problems and other conditions that often accompany obesity, such as type 2 diabetes.

Yet, according to Freedhoff, "we also know there is a precedent for people with obesity being systematically managed differently during a pandemic" - a potential factor in worse outcomes that research and media reports often overlook.

During the H1N1 pandemic a decade ago, people with obesity appeared to have a much higher risk of severe or fatal complications from infection - that is, until researchers took a closer look at the treatment they received.

As it turned out, "patients with obesity were less likely to receive early antiviral

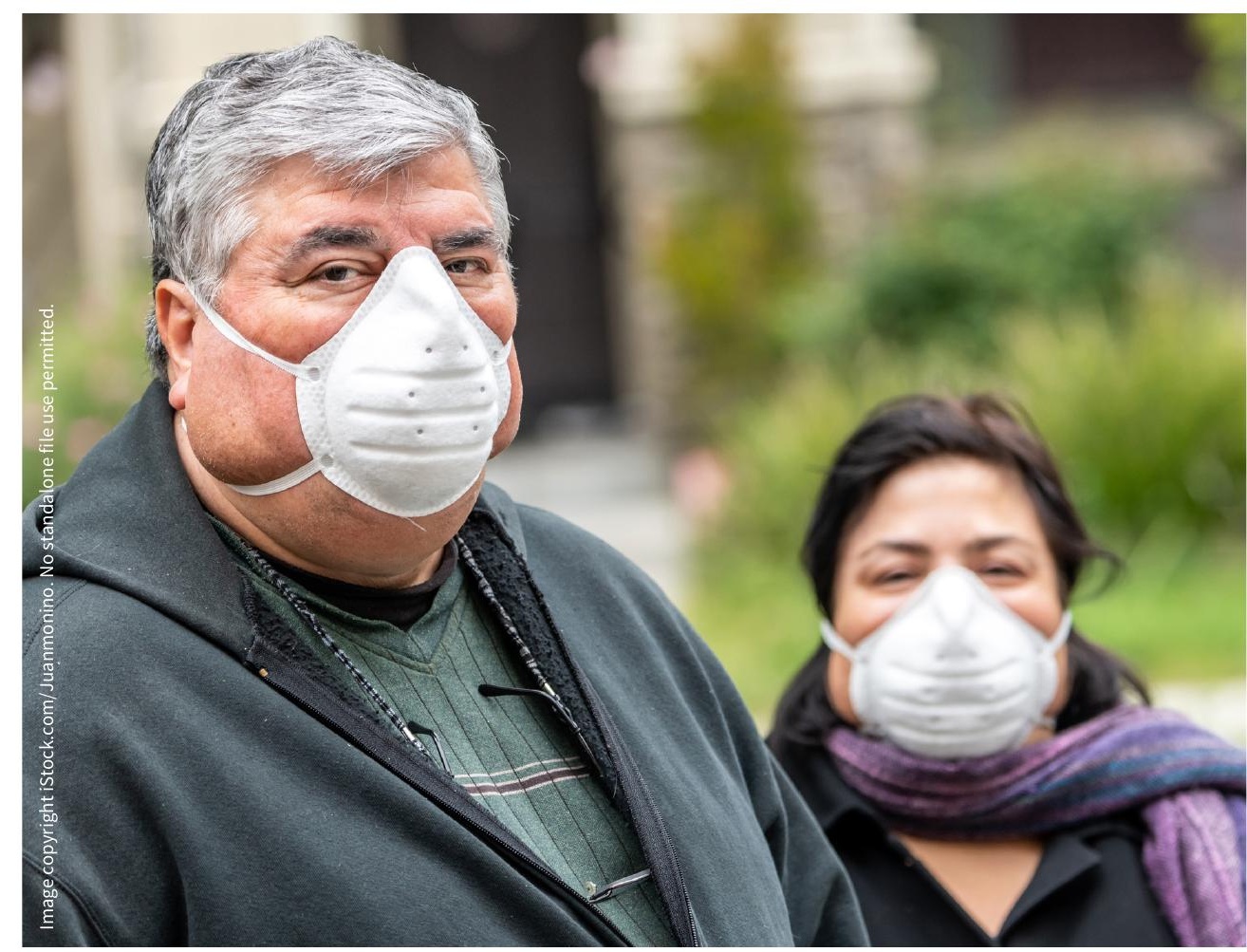

Weight bias and discrimination may be contributing to worse COVID-19 outcomes among heavier people.

treatment," Freedhoff explained. When researchers controlled for that disparity, the difference in complications "disappeared."

"People with obesity were discriminated against systematically, whether it was conscious, unconscious, implicit or explicit," Freedhoff said. "Could this be happening with COVID-19?"

People with higher BMIs who have faced weight bias or discrimination from health workers in the past may delay seeking care for symptoms of COVID-19, Freedhoff explained. Meanwhile, there's a dearth of information on the interventions and timeliness of the care they receive compared to other patients with COVID-19.
"I would love to see this more formally investigated because there is plausibility and precedent to suggest the possibility of bias," Freedhoff told CMAJ. "Did they receive steroids later? Were they proned less frequently? Were they admitted [to hospital or intensive care] in later stages of the disease?"

For example, "when it comes to people with severe obesity, proning might not have been completed at the same rate or at all" given staffing challenges and the number of people required to properly position patients with higher BMIs, Freedhoff said. Yet, patients with obesity and acute respiratory distress may benefit more from the intervention than patients with lower BMIs. 
Freedhoff also argued that media reports have overemphasized obesity as a risk factor for severe COVID-19 relative to other risks. He noted that people with a BMI between 30 and 35, also known as class I obesity, face comparable risks to those with chronic heart or lung disease, cancer and dementia, and lower risks than those associated with being older than 50, Black or South Asian.

According to Freedhoff, fear-mongering rhetoric does not help people and exacerbates the stigma that many already face. One study published in the International Journal of Obesity in 2015 found that news articles that frame fatness as negative increased anti-fat beliefs in readers.

"Yet, there is this constant call in the media that this is an obesity emergency, which in turn fuels weight bias," says Freedhoff. "It leads to articles suggesting this is an easy thing to fix, that it is the fault of individuals with obesity, which doesn't help anyone. And it's probably worth mentioning, if these scary articles do inspire people to actively lose weight ... there is no data at this point to suggest that it's any safer to be in an active phase of weight loss when facing COVID."

\section{Diana Duong, CMAJ}

Content licence: This is an Open Access article distributed in accordance with the terms of the Creative Commons Attribution (CC BY-NC-ND 4.0) licence, which permits use, distribution and reproduction in any medium, provided that the original publication is properly cited, the use is noncommercial (i.e., research or educational use), and no modifications or adaptations are made. See: https://creativecommons.org/ licenses/by-nc-nd/4.0/ 